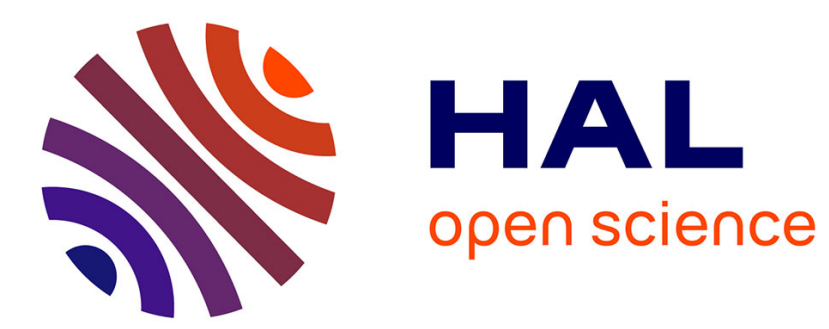

\title{
Solutions. Solubilité. II
}

E. Darmois

\section{- To cite this version:}

E. Darmois. Solutions. Solubilité. II. J. Phys. Radium, 1943, 4 (11), pp.233-245. 10.1051/jphysrad:01943004011023300 . jpa-00233850

\section{HAL Id: jpa-00233850 https://hal.science/jpa-00233850}

Submitted on 1 Jan 1943

HAL is a multi-disciplinary open access archive for the deposit and dissemination of scientific research documents, whether they are published or not. The documents may come from teaching and research institutions in France or abroad, or from public or private research centers.
L'archive ouverte pluridisciplinaire HAL, est destinée au dépôt et à la diffusion de documents scientifiques de niveau recherche, publiés ou non, émanant des établissements d'enseignement et de recherche français ou étrangers, des laboratoires publics ou privés. 


\title{
LE JOURNAL DE PHYSIQUE
}

$\mathbf{E} \mathbf{T}$

\section{LE RADIUM}

\section{SOLUTIONS. SOLUBILITÉ. II.}

\author{
PaI E. DARMOIS.
}

\begin{abstract}
Sommaire. - Un premier exposé a donné l'essentiel de la thermodynamique des solutions. On montre dans le présent exposé que la solubilité d'un gaz et celle d'un solide sont prévisibles quand la solution obéit à la loi de Raoult; celle d'un liquide est totale dans le même cas. On établit pour un solide l'équation de Schröder-Le Chatelier, qui permet de tracer la courbe de congélation d'un mélange, de calculer la composition et le point de fusion d'un eutectique. On traite le cas d'un corps dissous dissociable, des cristaux mixtes.

L'expérience n'est pas loujours d'accord avec la théorie; c'est parce que la loi de Raoult n'est qu'une loi-limite. Si l'on connaît la courbe des pressions de vapeur d'un composant, on peut prévoir sa solubilité. Les déviations par rapport à la loi de Raoult introduisent la notion d'activité selon Lewis. A cause de l'importance de cette notion et de la difficulté où l'on est actuellement de se procurer le livre de Lewvis et Randall, nous croyons utile d'indiquer succinctement les procédés de calcul de l'activité.

Le coefficient d'activité varie avec la concentration de la solution; quand la formule est simple, on peut prévoir certaines singularités, en particulier la démixtion, formation de deux couches liquides.
\end{abstract}

Comme nous l'avons vu dans un article précédent $\left({ }^{1}\right)$, la loi de Raoult relie la proportion d'une substance dans la phase gazeuse à sa proportion dans la phase liquide; elle permet donc de calculer la solubilité d'une vapeur dans une solution. Cette solubilité "idéale " est, dans certains cas, voisine de la solubilité réelle. Nous commencerons donc par la calculer, quitte à voir ensuite les différences entre le calcul et l'expérience et les raisons de ces écarts.

A. Solubilité des gaz. - Quand le gaz est au-dessous de sa température critique, c'est une vapeur et ce que nous avons dit précédemment s'applique. Pour certains gaz dont les pressions de saturation sont élevées, des corrections s'imposeraient pour tenir compte du fait que les lois des gaz parfaits ne s'appliquent plus. On peut généralement s'en dispenser car, en même temps, la loi de Raoult n'est plus qu'approchée.

Au-dessus de la température critique, on ne peut

(1) J. de Physique, 1943, 4, 129. Cette référence est notée (Exp. I) dans ce qui suit. Nous renvoyons à cet article pour les notations. plus parler de pression de vapeur, mais, dans le graphique de $\log p$ en fonction de $T$, on peut extrapoler jusqu'à la température de l'expérience. La figure I représente cette extrapolation pour li méthane à la température de $25^{\circ} \mathrm{C}$; la température critique est $-83^{\circ} \mathrm{C}$. On trouve à $2 \tilde{\partial}^{\circ} p_{0}=370 \mathrm{~atm}$. Si la pression du gaz est i atm au-dessus de la solution, le rapport molaire du corps dissous dans la solution est $x_{2}=\frac{p}{p_{0}}$, soit $\frac{\mathrm{I}}{370}=0,0027$. Ce nombre représenie la solubilité théorique du méthane dans un liquide quelconque sous la pression ordinaire. En dépit des approximations et extrapolations faites, c'est bien l'ordre de grandeur. Dans l'hexane, où la loi de Raoult s'applique, l'expérience donne o,oo3 I ; dans le xylène, on trouve 0,0026 .

Théoriquement, exprimée en rapport molaire, la solubilité est la même dans tous les liquides donnant des solutions idéales. Exprimée en concentration volumique, la solubilité est plus grande dans les solvants de faible volume molaire.

Si l'on change la pression du gaz au-dessus de la solution, $x_{2}$ varie proportionnellement à cette pression, c'est la loi de Henry.

Si l'on augmente $T, p_{0}$ augmente, la solubilité 
diminue; si l'extrapolation est toujours permise, la variation de $p_{0}$ peut être calculée par la formule de Clapeyron.

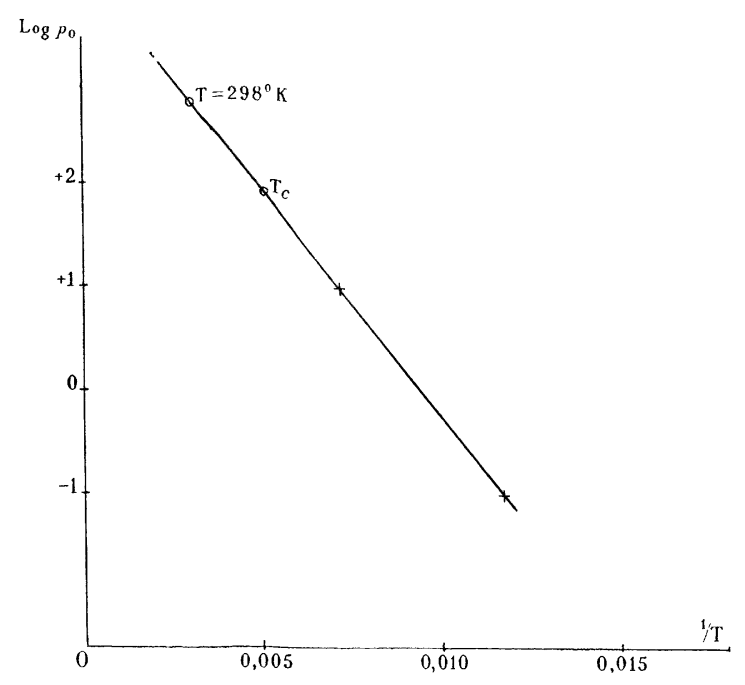

Fig. I .

Enfin, sous des pressions extérieures égaìes, le gaz le plus soluble sera celui dont la température critique est la plus élevée, $p_{0}$ ètant alors plus faible. Cette dernière règle est assez bien vérifiée, comme le montre le Tableau I donnant quelques solubilités dans le benzène à $20^{\circ} \mathrm{C}$.

Tableau I.

\begin{tabular}{|c|c|c|c|c|}
\hline Gaz. & $\mathrm{H}_{\mathrm{u}}$. & $\mathrm{N}_{2}$. & Co. & $\mathrm{CO}_{2}$ \\
\hline$T_{c} \ldots$ & 38 & 127 & 132 & $304^{\circ} \mathrm{K}$ \\
\hline$x_{2} \ldots$ & 0,026 & 0,0 年 & 0,06 I & o,9í \\
\hline
\end{tabular}

Nous reviendrons plus tard sur les solubilités réelles des gaz.

B. Solubilité des liquides. - Comme le corps dissous est à l'état liquide dans la solution, si celle-ci est idéale, elle est une simple juxtaposition des deux espèces moléculaires; les deux liquides sont donc miscibles en toute proportion et la solubilité est illimitée. On sait que, ce n'est pas toujours le cas, comme nous le reverrons également. Par exemple, on obtient souvent deux couches liquides superposées; c'est qu'alors le mélange ne suit pas la loi de Raoult. En effet, la pression de vapeur partielle du $n^{0} 1$ est forcément la mème dans les deux couches puisque c'est celle qui existe dans la vapeur. Si la loi de Raoult s'applique, le rapport molaire du no 1 est le même dans les deux couches; elles n'en forment donc qu'une.

Nous reviendrons plus loin sur la solubilité limitée des liquides; dès maintenant il est certain que, entre la solution idéale et la démixtion visible, il existera évidemment tous les intermédiaires.

C. Solubilité des solides; courbe de solubilité idéale. - A la saturation, la solution est en contact avec le solide. La pression partielle de celui-ci est donc égale à la pression de sublimation, sinon il n'y aurait pas équilibre; donc $p_{2}=p_{\text {.. }}$. D'autre part la pression de vapeur est aussi $p_{2}^{0} . x_{2}$ où $p_{2}^{0}$ est la pression de vapeur du no 2 pur, supposé à l'état liquide. Ce liquide ne serait pas stable à la température de l'expérience puisque la forme stable est le solide. Donc $p_{2}>p_{s}$ et la présence du solvant a précisément pour effet d'abaisser la pression de vapeur du liquide au niveau de celle du solide. La comparaison des deux relations précédentes donne de suite

$$
x_{2}=\frac{p_{s}}{p_{2}^{0}} .
$$

Cette relation très simple s'applique à l'apparition des cristaux du no 2 quand on refroidit un mélange homogène de $(2)$ et de $(1) ; x_{2}$ représente la solubilité du $\mathrm{n}^{\circ} 2$ dans le $\mathrm{n}^{\circ} 1$.

Pour connaître la solubilité, il faut donc calculer le rapport des pressions de saturation du solide et du liquide à la même température. Ce calcul est possible en utilisant la formule de Clapeyron. Nous emploierons d'abord les notations classiques en France.

Pour le liquide, on a

$$
\frac{\mathrm{d}}{\mathrm{d} T} \ln p_{2}^{0}=\frac{J L_{v}}{R T^{2}}
$$

où $L_{v}$, est la chaleur de vaporisation molaire; on a supposé comme d'usage le volume spécifique du liquide négligeable vis-à-vis de celui de la vapeur, celle-ci suivant l'équation des gaz parfaits.

Pour le solide, on a de même

$$
\frac{\mathrm{d}}{\mathrm{d} T} \ln p,=\frac{J L_{s}}{R T^{2}}
$$

où $L$, est la chaleur de sublimation, avec les mêmes approximations.

En retranchant les deux équations précédentes, il vient

$$
\frac{\mathrm{d}}{\mathrm{d} T} \ln x_{2}=\frac{J L f}{R T^{2}}
$$

où $L$, est la chaleur de fusion.

Cette équation peut aussi s'écrire avec les notations employées précédemment (Exp. I). L'équation (14) (Exp. I) réglait la variation de la fugacité partielle d'un composant; c'est la généralisation de la formule de Clapeyron. En retranchant l'une de 
l'autre les deux équations analogues pour le liquide et le solide, on obtient

$$
\left[\frac{\mathrm{d}}{\mathrm{d} T} \ln x_{2}\right]_{/,}=\frac{\left(\overline{H_{2}}-H_{2}^{\prime}\right)}{R T^{2}}=\frac{\Delta I I}{R T^{2}},
$$

où $\Delta H$ est la chaleur différentielle de dissolution. Comme la solution est idéale, et que le corps dissous est à l'état liquide, c'est donc aussi la chaleur de fusion du corps dissous supposé solide.

L'équation (2) a d'abord été obtenue par Schröder [r], puis indépendamment par Le Chatelier [2].

Pour utiliser cette équation, il est nécessaire de l'intégrer. Pour cela on peut supposer d'abord que la chaleur de fusion est constante et indépendante de $T$. La constante d'intégration se détermine en faisant $T=T_{f}\left({ }^{2}\right)$; on a alors $x_{2}=$ I ; c'est l'équilibre du liquide $\mathrm{n}^{0} 2$ en présence du solide. On trouve de suite

$$
\log _{10}\left(\frac{1}{x_{2}}\right)=\frac{L_{f}}{4,57}\left(\frac{1}{T}-\frac{1}{T_{f}}\right) .
$$

Si l'on veut tenir compte de la variation de $L_{f}$ avec $T$, on écrira l'équation de Kirchhoff

$$
\frac{\mathrm{d} L_{f}}{\mathrm{~d} T}=C_{l}-C_{,}
$$

où les $(C)$ sont les chaleurs spécifiques molaires du liquide et du solide. En les supposant de nouveau indépendantes de $T$, on trouve finalement

$$
\begin{aligned}
\log \left(\frac{1}{x_{2}}\right)=\frac{L_{f}^{0}\left(T_{f}-T\right)}{4,57 T T_{f}} & -\frac{C_{l}-C_{s}}{4,57} \frac{T_{f}-T}{T} \\
& +\frac{C_{l}-C_{s}}{1,985} \log \frac{T_{f}}{T},
\end{aligned}
$$

$L^{0}$, est la chaleur de fusion au point de fusion normal. Les deux formules (3) et (4) représentent avec deux approximations différentes la courbe de solubilité du no 2 dans le solvant $n^{\circ} 1$ ou encore la courbe de congélation du mélange homogène des deux composants.

Schröder a déjà indiqué des vérifications de ces formules pour la solubilité de la naphtaline et des corps analogues. Pour $\mathrm{C}_{10} \mathrm{H}_{8}$, on a $L_{j}^{0}=4440$ cal; $C_{l}=56,6 ; C_{\varsigma}=5 \mathrm{r}, 8$ à $T_{j}^{\prime \prime}$. Pour $T_{f}^{0}=298$, I $\left(25^{\circ} \mathrm{C}\right)$ et $T=353^{\circ}, \mathrm{r} 5$, on trouve $x_{2}=0,3 \mathrm{I}$ par l'équation (3) et o,32 par l'équation (4). C'est ia solubilité idéale de $\mathrm{C}_{10} \mathrm{H}_{8}$ dans n'importe quel solvant. Schröder a vérifié que $\mathrm{C}_{10} \mathrm{H}_{8}$ se dissout à peu près de même dans $\mathrm{C}_{6} \mathrm{H}_{6}, \mathrm{C}_{6} \mathrm{H}_{5} \mathrm{Cl}, \mathrm{CCl}_{4}$, En portant en ordonnée $\frac{\mathrm{I}}{\bar{T}}$ et en abscisse $\log x_{2}$, on doit avoir une droite. La figure 2 représente $\left({ }^{3}\right)$ les courbes de congélation de $\mathrm{C}_{10} \mathrm{H}_{8}$ en présence de diverses impuretés. La droite idéale est effectivement suivie

( $\left.{ }^{2}\right)$ Plus exactement, $T$ est égale à la température du point triple où $p_{s}=p_{2}^{0}$.

$\left({ }^{3}\right)$ D'après Hildebrand, Solubility, New-York, 1936. pour $\mathrm{C}_{6} \mathrm{H}_{5} \mathrm{Cl}$ et la diphénylamine; son coefficient angulaire est 4,57. Dans les liquides qui lui ressemblent chimiquement, comme le benzène, le naphtalène suit assez convenablement la loi de Raoult;

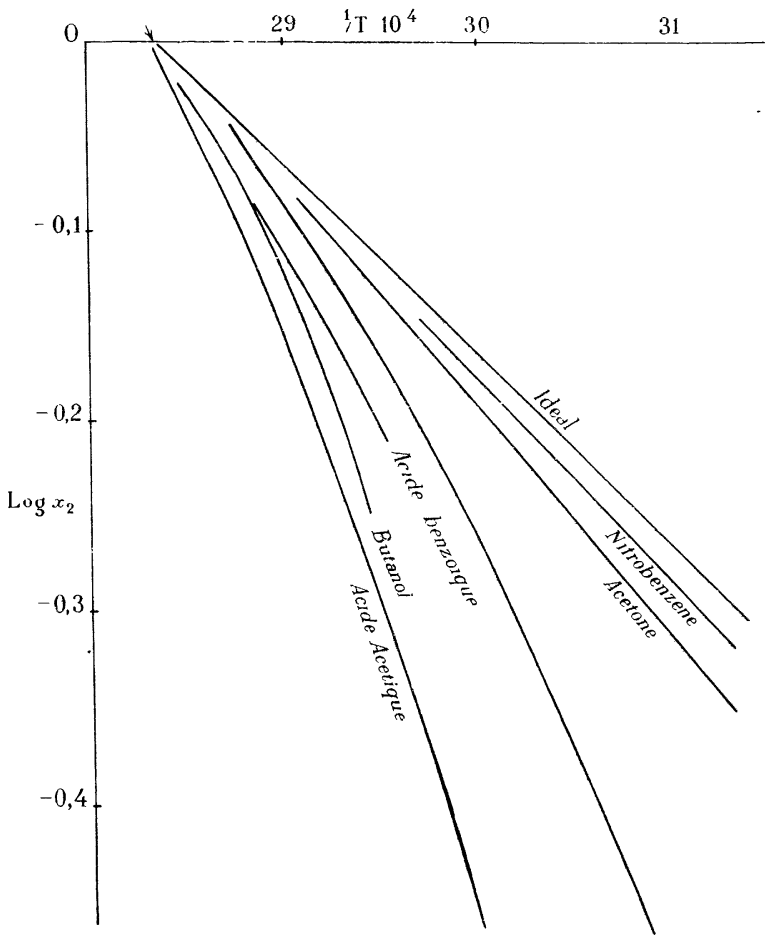

Fig. 2.

la solubilité dans $\mathrm{C}_{6} \mathrm{H}_{6}$ est 0,29 , très voisine de celle calculée. La flèche indique le point de fusion du naphtalène.

Schröder a aussi vérifié par exemple que la courbe

\begin{tabular}{|c|c|c|}
\hline P. F. $\left({ }^{\circ} \mathrm{C}\right)$. & $L_{f}=4000$ & $L_{f}=7000$ \\
\hline jo. . & $x_{2}=0, \dot{j} y^{3}$ & $x_{2}=0,401$ \\
\hline I0o....... & $o, 259$ & o,o9㣙 \\
\hline 200. & o,o833 & 0,0129 \\
\hline joo........ & 0,0158 & $0,0007 \mathrm{I}$ \\
\hline I00o........ & $0,00 \%$ & 0,00012 \\
\hline
\end{tabular}
de solubilité (ou de congélation) idéale de $p . \mathrm{C}_{6} \mathrm{H}_{4} \mathrm{Br}_{2}$ est à peu près la même dans $\mathrm{C}_{6} \mathrm{H}_{6}, \mathrm{CS}_{2}, \mathrm{C}_{6} \mathrm{H}_{5} \mathrm{Br}$, mais pas dans l'éthanol.

Tableau II.

Les chaleurs de fusion des corps normaux sont peu variables; elles sont de l'ordre de 5 ooo $\mathrm{cal} / \mathrm{mol}$; mais les points de fusion sont très variables; dans la formule (3), c'est donc $T_{J}$ qui déterminera les 
grosses variations de la solubilité. Le Tableau II donne par exemple les solubilités théoriques pour des substances dont les $\mathrm{P}$. F. varient de $50^{\circ}$ à $\mathrm{I} 000^{\circ} \mathrm{C}$ et pour deux chaleurs de fusion : 4000 et 7000 cal.

Certaines solubilités suivent bien la règle soulignée ci-dessus. Exemple $m$. o. et $p . \mathrm{C}_{6} \mathrm{H}_{4}\left(\mathrm{NO}_{2}\right)_{2}$ dans $\mathrm{C}_{6} \mathrm{H}_{6}$ à $50^{\circ} \mathrm{C}\left({ }^{4}\right)$.

$$
\begin{array}{rc}
\text { P. F. }\left({ }^{\circ} \mathrm{C}\right) . & x_{2 .} \\
90 \ldots \ldots \ldots \ldots & 0,376 \\
1 \mathrm{1} 6 \ldots \ldots \ldots \ldots & 0, \mathrm{I} ;> \\
170 \ldots \ldots \ldots \ldots & 0,03 \mathrm{I}
\end{array}
$$

La loi idéale (3) donne la solubilité par le rapport molaire du corps dissous. Pour passer du rapport $x_{2}$ au titre, il faut connaître le poids moléculaire des deux composants et en particulier celui du solvant. Pour les solvants organiques comme le benzène, l'emploi du P. M. chimique donne des résultats corrects, comme on l'a vu par les nombres ci-dessus.

Quand il s'agit d'un corps anormal, comme l'eau, la formule (3) donne des résultats inexacts en prenant is comme P. M. de l'eau. Par exemple, la solubilité de la naphtaline dans l'eau est environ $\mathrm{o}, \mathrm{I} \mathrm{g} / \mathrm{l}$, ce qui donne

$$
n_{2}=\frac{0,1}{128} \quad \text { el } \quad n_{1}=\frac{\mathrm{I} 000}{\mathrm{I} 8}=55,5 ;
$$

$x_{2}$ exp. est donc seulement o, oooor4. Certains auteurs ont vu dans cette discordance une preuve de la polymérisation de l'eau. On peut en effet raisonner comme suit.

Supposons l'eau polymérisée dans le rapport $q$; on a toujours $n_{2}=\frac{\mathrm{o}, \mathrm{I}}{\mathrm{I} 28}$, mais $n_{1}=\frac{\mathrm{J} 000}{\mathrm{I} 8} \cdot$ En écrivant que le rapport molaire du naphtalène est égal au rapport idéal 0,3 I, on trouve $q=32$ ooo. On sait qu'on préfère admettre maintenant une structure quasi réticulaire pour l'eau. A la rigueur le résultat ci-dessus est compatible avec cette structure.

Le nombre "idéal " pour la solubilité de $\mathrm{CINa}$ est $x_{2}=$ o, ooo 5 environ $\left(L_{f}=7220\right.$ et $\left.T_{f}=1077^{\circ} \mathrm{K}\right)$. Ce nombre est nettement inférieur à la solubilité mesurée de ClNa dans l'eau : 36 I g pour ı ooo g d'eau; ici il est impossible de rendre compte de la discordance en invoquant une polymérisation de l'eau. Les électrolytes se distinguent nettement des non électrolytes.

Dans la formule (3), on voit de suite que la solubilité augmente avec $T$; c'est, comme on sait, le cas pour tous les corps normaux.

Calcul de la température D'eutexie. - Quand on connaît la chaleur de fusion d'un corps, on peut prévoir sa cuurbe de congéiation idéale, c'est-à-dire sa solubilité dans un solvant idéal. Si l'on connâ̂t

(4) Cette variation de la solubilité avec le P. F. du corps dissous avait déjà été remarquée par Lavoisıer, Traité élémentaire de Chimie, 1789. la chaleur de fusion des deux constituants d'une solution, on doit pouvoir calculer leur solubilité réciproque, c'est-à-dire prévoir la température d'eutexie. C'est ce qui a été fait par Washburn et Read [3].

On a

$$
-\log x_{1}=\frac{L_{1}}{4,5 ;}\left(\frac{1}{T}--\frac{1}{T_{1}}\right)
$$

et

$$
-\log x_{2}=\frac{L_{2}}{1, j ;}\left(\frac{1}{T}-\frac{1}{T_{2}}\right)
$$

Pour une valeur de $T$ donnée, chaque formule donne $x_{1}$ et $x_{2}$ et en général $x_{1}+x_{2}$ est différent de l'unité. Au point d'eutexie, la même valeur de $T$ doit correspondre à deux valeurs dont la somme est г. En posant $\frac{\mathrm{I}}{T}=\theta$ et $\frac{L}{1,57}=l$, la condition est

$$
10 l_{1}(\theta-1)+10 l_{2}(1)-\left(\theta_{2}\right)=1 .
$$

Le calcul de $\theta$ s'effectue par approximations successives; il est d'ailleurs aussi simple d'opérer directement sur les formules en log et de comparer les valeurs des $(x)$.

Pour $\mathrm{C}_{10} \mathrm{H}_{8}$ et $\mathrm{C}_{6} \mathrm{H}_{6}$, les valeurs des $(L)$ sont 4570 et 2370 , celles des $(l)$ rooo et 5 r 9 , celles des $(T) 353,3$ et 278,7 , celles des (0) 0,00283 et o,oo359. Le calcul donne $0=0,0037 \mathrm{I}, T=269,7$, c'est-à-dire $t=-30,55$. L'observation donne $-3^{\circ}, 4^{8}$. On a des concordances du même ordre pour les deux couples suivants :

$$
\begin{array}{lcc} 
& \text { Calculé. } & \text { Observé. } \\
\text { Benzène-diphıényle........... } & -6,1 & -j, 8 \\
\text { Diphıényle-naphtalène..... } & 39, i & 39, i
\end{array}
$$

Courbe de SOlubilité D'un corps Dissous Dissociable. - Pour établir la formule (3), nous avons supposé que le corps solide de fond est identique au liquide $n^{\circ} 2$; cela exclut la formation de cristaux mixtes ou le cas où le corps fondu se dissocie. Nous traiterons d'abord ce dernier cas; il est assez répandu $\left({ }^{5}\right)$; il correspond aux courbes de congélation avec maximum. La courbe de solubilité du corps présente un maximum pour lequel la composition du liquide et celle du solide de fond sont les mêmes. La figure 3 se rapporte au mélange $\left.\mathrm{C}_{2} \mathrm{H}_{2}+\left(\mathrm{CH}_{3}\right)_{2} \mathrm{O}{ }^{6}{ }^{6}\right)$. Le système est binaire, mais une partie des constituants est combinée sous la forme $A$. $p B$. Le système peut être considéré comme

(5) Nous avons par exemple relevé dans Timmermans (Solutions concentrées, Masson, 1936) la présence de courbes de congélation avec maximum pour les mélanges

$$
\mathrm{C}_{2} \mathrm{H}_{4} \mathrm{Cl}_{2}+\mathrm{C}_{2} \mathrm{H}_{2} \mathrm{Cl}_{4} ; \quad \mathrm{CH} \mathrm{Cl}{ }_{3}+\left(\mathrm{CH}_{3}\right)_{2} \mathrm{O} ;
$$

aldéhyde + éthanol; aniline + phénol, etc. C'est aussi le cas des nombreux hydrates (ou solvates) tels que $\mathrm{Cl}_{2} \mathrm{Ca} .6 \mathrm{H}_{2} \mathrm{O}$.

${ }^{(6)} \mathrm{Si}$ la combinaison était complète, la courbe du milieu serait composée de deux droites se coupant à angle vif audessus du maximum de la courbe réelle. On peut dire déjà que ce sont les produits de décomposition qui abaissent le P. F. du corps pur. 
formé à l'aide de $n_{1}$ moles de $A, n_{2}$ de $B, n_{3}$ du composé $A . p B$.

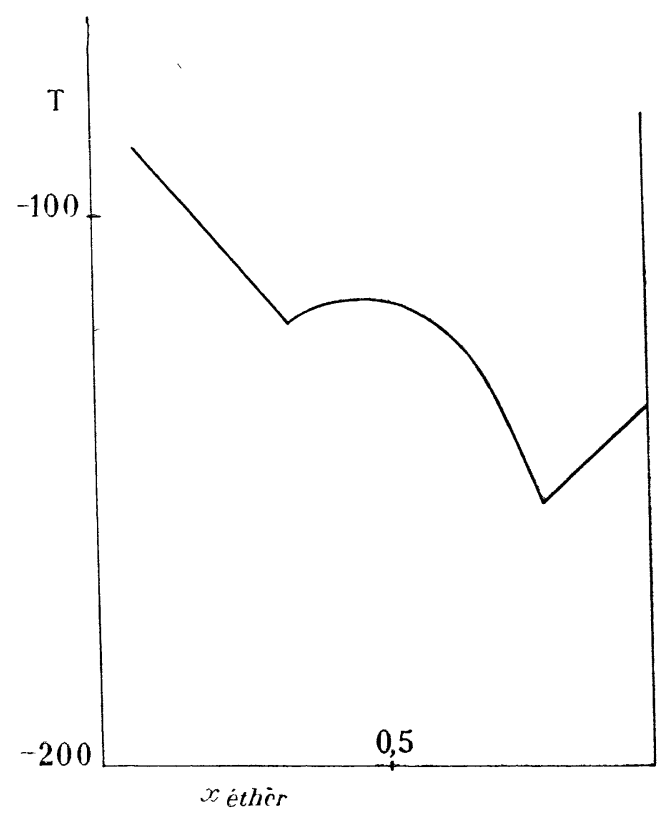

Fig. 3.

Au point de fusion de ce composé, on a $n_{2}=p n_{1}$ Supposons qu'on ajoute $\mathrm{d} n_{1}$ moles de $A$. La formule de Duhem-Margules s'écrit

$$
x_{1}\left(\frac{\partial \overline{F_{1}}}{\partial x_{1}}\right)+x_{2}\left(\frac{\partial \overline{F_{2}}}{\partial x_{2}}\right)+x_{3}\left(\frac{\partial \overline{F_{3}}}{\partial x_{3}}\right)=0 .
$$

Elle donne ici

$$
n_{1} \mathrm{~d} \overline{F_{1}}+n_{2} \mathrm{~d} \overline{F_{2}}+n_{3} \mathrm{~d} \overline{F_{3}}=0
$$

ou

$$
n_{1}\left(\mathrm{~d} \bar{F}_{1}+p \mathrm{~d} \bar{F}_{ \pm}\right)+n_{3} \mathrm{~d} \overline{\boldsymbol{F}}_{3}=0 .
$$

Comme les trois substances sont en équilibre,

$$
\Delta F=o=F_{3}-p F_{2}-F_{1} .
$$

D'où

$$
\mathrm{d} \overline{F_{3}}=\mathrm{d} \bar{F}_{1}+p \mathrm{~d} \overline{F_{2}} .
$$

En comparant (5) et (6), on a

$$
\left(n_{1}+n_{i ;}\right) \mathrm{d} \overline{F_{3}}=0, \quad \text { c'est-à-dire } \quad \widehat{\mathrm{d}} \overline{F_{3}}=0 .
$$

La fugacité du composé ne change donc pas par addition d'une petite quantité d'un de ses compo sants; le composé reste en équilibre avec le solide à température constante.

Le calcul précédent ne donne aucun renseignement sur l'étendue de la dissociation en solution. Deux méthodes ont été proposées pour évaluer cette dissociation,
MÉthode de Kremann [4]. - Elle utilise l'abaissement cryoscopique dans la combinaison additionnelle fondue; si l'on dissout dans celle-ci un corps qui n'est pas l'un des constituants, l'abaissement observé donne la constante cryoscopique. Si l'on ajoute un des constituants, $A$ par exemple, l'équilibre $A+p B \rightleftharpoons A B_{p}$ se déplace; une partie de $A$ se combine, donc ne produit pas d'abaissement. On admet que l'équilibre obéit à la loi d'action de masses et, moyennant un certain nombre d'hypothèses, on peut calculer le degré de dissociation.

Kremann étudie par exemple la combinaison additionnelle aniline-m-crésol. Son point de fusion à l'état pur est - $14^{\circ}, 2$. En y dissolvant du benzène ou de l'acétate d'éthyle, il détermine l'abaissement cryoscopique normal; on trouve ainsi un abaissement $\Delta=0^{\circ}, 28$ pour la dissolution de $\mathrm{I}$ mol dans roo au total. On ajoute successivement les deux composants de la combinaison, à savoir le $m$-crésol et l'aniline. On constate d'abord que l'abaissement est le même, quel que soit le composant ajouté, pourvu que sa fraction molaire soit la même. On obtient ainsi les nombres suivants :

Fraction molair'e de l'addition... $0,05 \quad 0,10 \quad 0,15$ Ibaissement $\left({ }^{\circ}\right) \ldots \ldots \ldots \ldots \ldots \ldots, 0,95 \quad 1,00 \quad 2,00$

La dissociation du composé est du type $A B \rightleftharpoons A+B$.

Sur roo moles du composé, soit $\alpha$ la fraction décomposée pour le corps pur; il reste Iо० $(\mathrm{I}-\alpha)$ moles et il apparaît $200 \alpha$ moles de $A$ et $B$; on a donc en tout roo + roo $\alpha$ moles. Si l'on ajoute en plus $a$ moles de $A$, le nombre total des moles peut s'écrire $100+x+a$, où $x$ est le nombre de moles décomposées dans les roo moles primitives. Si $K$ est la constante de dissociation, la loi d'action de masses s'écrit :

$$
\frac{x}{100+x+a} \frac{x+a}{100+x+a}=K \frac{100-x}{100+x+a} .
$$

On ne connaît ni $x$, ni $K$; on procède donc par tâtonnements. On se fixe un degré de dissociation, soit $\alpha=0$, 10. Pour $a=0$, on a alors $x^{2}=K\left(\mathrm{ro}^{4}-x^{2}\right)$, avec $x=\mathrm{Io}$, d'où $K=$ o, огог. Le nombre $y$ de moles produisant l'abaissement, dans ioo moles au total, est alors $\frac{20}{1 \text { IO }}=18,18$. On donnera ensuite à $a$ des valeurs croissantes, en admettant que $K$ reste constant, ce qui donne par exemple l'ensemble de nombres suivants. $\delta$ est l'abaissement calculé; pour $a=6$, on a

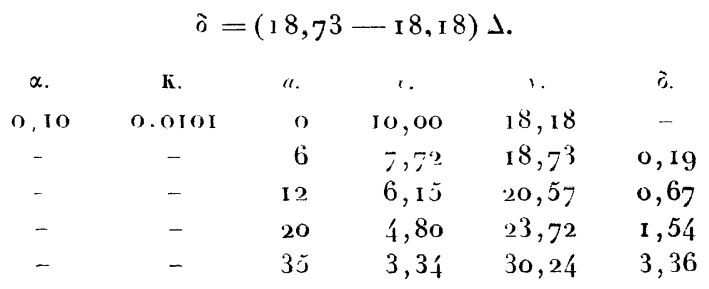


On construit la courbe des ò en fonction de $a$ ou du rapport molaire de l'addition et l'on cherche la valeur de $\alpha$ qui assure la meilleure concordance entre la courbe calculée et la courbe expérimentale. Van Laar a d'ailleurs proposé [5] une formule qui tient compte de la dissociation ciu solvant crvoscopique; elle s'écrit :

$$
-\ln \frac{\mathrm{I}+\alpha_{0}(\mathrm{I}-\alpha)(\mathrm{I}-x)}{\mathrm{I}-\alpha_{0}(\mathrm{I}+\alpha)(\mathrm{I}-x)}=\frac{L_{f}}{R}\left(\frac{\mathrm{I}}{T}-\frac{\mathrm{I}}{T_{f}}\right)
$$

$\alpha_{0}$ est le degré de dissociation à l'état pur, $\alpha$ le même en présence d'une fraction molaire $x$ du deuxième composant. Avec $L_{f}=3900$ calories, on obtient $\operatorname{des} \alpha_{0}$ et $\alpha$ voisins de o, ı o. C'est à peu près ce qu'avait donné le calcul de Kremann.

Les calculs cryoscopiques sont en réalité plus compliqués que ceux de Kremann et ils ne peuvent donner que l'activité de la substance dissoute, dès que la solution devient tant soit peu concentrée. Les calculs de Kremann sont donc basés sur des hypothèses assez douteuses.

Méthode de Job [6]. - Elle repose aussi. sur un certain nombre d'hypothèses; mais elle a l'avantage de renseigner sur la formule du composé et en même temps sur la constante de dissociation, c'est-à-dire sur la stabilité du composé. Un grand nombre de travaux effectués dans mon laboratoire ont utilisé cette méthode de Job. Il se trouve qu'elle a été jusqu'ici presque exclusivement employée pour les solutions d'électrolytes dans l'eau. Nous en reparlerons plus tard.

Solubilité (CONGÉlation) DANS LE CAS OU IL SE FORME DES CRISTAUX MIXTES. - On rencontre ce cas très fréquemment à haute température; le corps dissous est soluble dans la phase solide du solvant. Soient $x_{1}$ et $x_{2}$ les rapports molaires dans la solution, $x_{1}^{\prime}$ et $x_{2}^{\prime}$ dans la solution solide. Cette fois la fugacité du solide dépend de $T$ et des $\left(x^{\prime}\right)$. L'équilibre exige que $f_{1}^{\prime}$ (solide) $-f_{1}$ (liquide). Quand on ajoute du soluté, $\mathrm{d} f_{1}^{\prime}=\mathrm{d} f_{1}$; cela donne

$$
\begin{aligned}
& \left(\frac{\mathrm{d} \ln f_{1}^{\prime}}{\mathrm{d} T}\right)_{x_{2}^{\prime}, p}+\left(\frac{\mathrm{d} \ln f_{1}^{\prime}}{\mathrm{d} x_{2}^{\prime}}\right)_{T} \mathrm{~d} x_{2}^{\prime} \\
= & \left(\frac{\mathrm{d} \ln f_{1}}{\mathrm{~d} t}\right)_{x_{2}, p}+\left(\frac{\mathrm{d} \ln f_{1}}{\mathrm{~d} x_{2}}\right)_{T} \mathrm{~d} x_{2} .
\end{aligned}
$$

On suppose les deux solutions, liquide et solide, infiniment diluées en le no 2 .

D'après les formules de l'article sur la thermodynamique des solutions, on a

$$
\left(\frac{\mathrm{d} \ln f_{1}^{\prime}}{\mathrm{d} T}\right)_{x_{3}^{\prime}}=\frac{H_{1}^{\prime 0}-\overline{H_{1}^{\prime}}}{R T^{2}}
$$

et l'analogue pour $x_{2}$.

L'hypothèse soulignée permet d'écrire la loi de Raoult $\frac{d f_{1}^{\prime}}{\overline{d x_{e}^{\prime}}}=-f_{1}$. Il reste alors

$$
\frac{H_{1}^{\prime 0}-\overline{H_{1}^{\prime}}}{R T^{2}} \mathrm{~d} T-\mathrm{d} x_{2}^{\prime}=\frac{H_{1}^{\prime}{ }^{0}-\overline{H_{1}}}{R T^{2}} \mathrm{~d} T-\mathrm{d} x_{2},
$$

c'est-à-dire

$$
\mathrm{d} x_{2}-\mathrm{d} x_{2}^{\prime}=\frac{\overline{H_{1}^{\prime}}-\overline{H_{1}}}{R T^{2}} \mathrm{~d} T .
$$

On utilise ensuite la loi de répartition

$$
\mathrm{d} \frac{x_{2}^{\prime}}{\mathrm{d} \overline{x_{2}}}=\frac{k}{k_{1}^{\prime}} \text {. }
$$

Ce qui donne enfin

$$
\frac{\mathrm{d} T}{\mathrm{~d} \cdot x_{2}}=\left(\frac{k}{k_{1}^{\prime}}-1\right) \frac{R T^{\prime 2}}{\overline{H_{1}}-\overline{H_{1}^{\prime}}}
$$

$\bar{H}_{1}-\bar{H}_{1}^{\prime}$ concerne le passage du solide à la solution et si celle-ci est diluée (idéale), c'est l'enthalpie de fusion du solvant pur.

La thermodynamique ne donne aucun renseignement sur le domaine de validité de la formule (7).

\section{Discordances entre la théorie et l'expérience.}

A. Remarques qualitatives. - S'il y a quelques solubilités qui coïncident avec la solubilité idéale, il y a aussi beaucoup de discordances entre la théorie

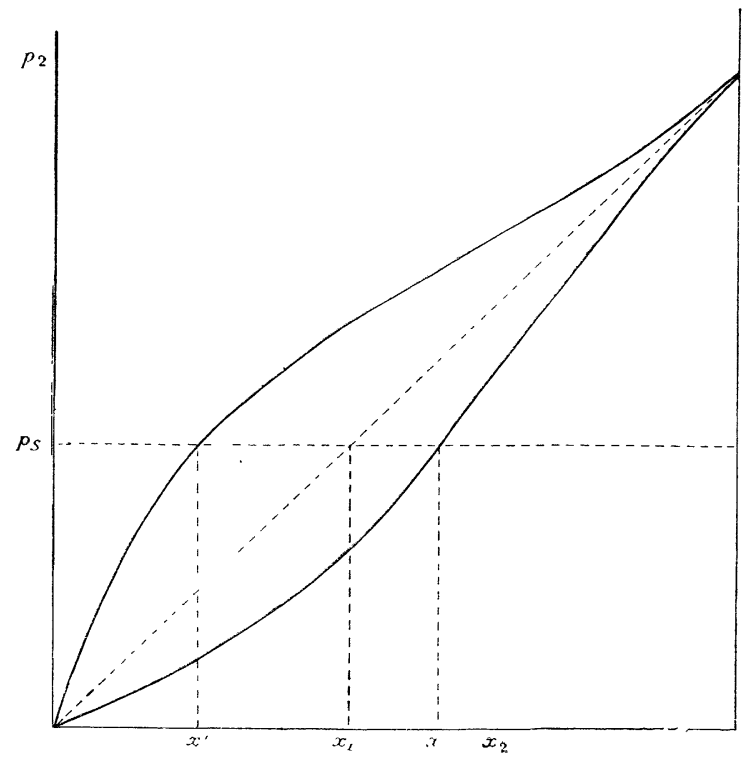

Fig. 4.

et l'expérience. Elles s'expliquent simplement par le fait que la courbe des pressions partielles n'est pas en général la droite exigée par la loi de Raoult. La pression de vapeur peut être supérieure à l'ordonnée de cette droite; la déviation est dite positive; c'est le cas du mélange $\mathrm{CS}_{2}-\mathrm{CH}_{2}\left(\mathrm{OCH}_{3}\right)_{2}$. La pression peut être inférieure à l'ordonnée de la droite; la 
déviation est dite négative; c'est le cas du mélange acétone-chloroforme. Enfin, dans tout le domaine étudié, la déviation peut changer de signe; c'est le cas pour la pyridine dans ses mélanges avec l'eau. Il faut mettre tout à fait à part les solutions électrolytiques, qui ne suivent pas la loi de Raoult.

Une fois la courbe des pressions partielles connue, on peut toujours utiliser la relation $p_{2}=p_{\text {s }}$ qui est toujours valable; on arrive ainsi aux résultats de la figure / où l'indice ? désigne le corps dissous. On a représenté sur cette figure la droite théorique en pointillé, les deux courbes positive et négative en trait plein. L'ordonnée de hauteur $p_{s}$ coupe les courbes en trois points dont les abscisses $x^{\prime}, x_{l}$ et $x^{\prime \prime}$ représentent le rapport molaire des corps dissous dans les trois cas. On voit que les déviations positives correspondent à des solubilités inférieures à la solubilité idéale, les négatives à des solubilités supérieures. En tout cas, la courbe des pressions partielles de vapeur étant connue, la solubilité s'en déduit de suite. Il faut maintenant se préoccuper : ${ }^{\circ}{ }^{0}$ de chiffrer les déviations; ${ }^{\circ}{ }^{\circ}$ d'en proposer une explication. Dans cet article, nous traiterons seulement le premier point, nous réservant de traiter le deuxième plus tard.

B. Grandeur des déviations de la loi de Raoult. - D'après la définition que nous avons donnée au premier article de cette série, pour le coefficient d'activité, la relation $\gamma=\frac{a}{x}$ montre que $\gamma$ est précisément la mesure des déviations. L'expression $R T \ln \gamma$ est le changement d'énergie libre dans le transfert d'une mole du composant, d'une quantité considérable de solution idéale à la solution réelle de même composition.

Tous les procédés dérivés de la loi de Raoult (tonométrie, ébullioscopie, cryoscopie, etc.) peuvent être employés en principe pour évaluer le coefficient d'activité. Les méthodes de calcul sont dues à Lewis; nous donnerons seulement ici quelques indications, renvoyant le lecteur à la thermodynamique de Lewis et Randall pour les détails.

Activité mesurée A partir de la pression de vapeur DU SOLVANT. - C'est le cas le plus simple. Si la vapeur est un gaz parfait, on a simplement $a=\frac{p_{1}}{p_{1}^{0}}$ C'est ainsi par exemple que Hildebrand et Eastman [7] évaluent l'activité du mercure dans les amalgames de thallium à $325^{\circ} \mathrm{C}$. Le Tableau III donne, d'après ces auteurs, l'activité et le coefficient d'activité de $\mathrm{Hg}$.

Activité DU CORPS Dissous A PARTIR DE SA PRESSION DE VAPEUR. - L'exemple est emprunté à Lewis et Storch [8] et concerne les solutions de brome dans $\mathrm{C} \mathrm{Cl}_{4}$. Les auteurs prennent comme état standard du brome celui en solution infiniment diluée; dans cet état, d'après la loi de Henry, $\frac{t_{2}}{p_{2}}$ est constant, soit $k$. Comme dans cet état, par définition, $a_{2}^{0}=x_{2}^{\prime \prime}$, à une concentration quelconque, on aura donc $a_{2}=p_{2}$. Les auteurs ont ainsi étudié les solutions entre les concentrations $x_{2}=0,0 o_{1}^{\prime}$ et 0,025 et ils ont trouvé un rapport $\frac{l_{2}}{r_{2}}$ constant et égal à 0,539 ( $p$ en atm) (P. M. du brome pris égal à r6o). Dans tout cet intervalle, la solution est donc idéale. Le brome n'y est d'ailleurs pas dans le même état que dans le liquide pur, car sa pression de vapeur est seulement o,280 atm à l'état pur; l'extrapolation donnerait au contraire o, J39 atm. Si la vapeur du corps dissous ne suit pas les lois des gaz parfaits, on introduira facilement la fugacité.

TABLEAU III.

\begin{tabular}{|c|c|c|}
\hline$x_{1}$. & $\sigma_{1}$. & $r_{1}$. \\
\hline $0,9 j$ & 0,935 & $0,99^{\circ}$ \\
\hline 0,942 & 0,938 & 0,996 \\
\hline 0,915 & $o, 90 \mathrm{I}$ & 0.985 \\
\hline $0,89^{3}$ & 0.875 & 0.980 \\
\hline o, 836 & 0.803 & $0,96 \mathrm{I}$ \\
\hline 0,742 & 0,690 & 0,930 \\
\hline o,66' & $0,60^{\circ}$ & 0,907 \\
\hline 0,614 & o, 每 & $o, 893$ \\
\hline o, 497 & o. $\{3\}$ & $0,8>\mathrm{I}$ \\
\hline 0,3 47 & $0,99^{3}$ & o,841 \\
\hline $0, ? 0 ?$ & o. I 66 & $0,8 \cdot$, \\
\hline
\end{tabular}

Activité a partir des Forces Électromotrices. - Le procédé a été beaucoup employé pour les amalgames. Par exemple, deux électrodes constituées par des amalgames de thallium, de compositions différentes, plongent dans une même solution électrolytique d'un sel thalleux. Quand la pile fonctionne, si $T l$ est le métal électrométriquement actif, l'un des amalgames s'enrichit aux dépens de l'autre. La f. é. m. de la pile est donnée par la formule

$$
\Delta F=R T \ln \frac{a_{2}}{a_{2}^{\prime}}=-N F \%
$$

Les (a) sont les activités de $T l$ dans les deux électrodes, $N$ est la valence du métal, $\mathscr{H}$ le Faraday. Avec les valeurs des constantes, faisant $N=$ r, il vient

$$
\left.E=-0,00019^{8} 1\right\} T \log _{10}\left(\frac{a_{2}}{a_{2}^{\prime}}\right) \quad(E \text { en volts }) .
$$

Richards et Daniels [9] ont effectué des mesures à $20^{\circ} \mathrm{C}$; on a alors

$$
\log a_{2}=-\frac{E}{0,05816}+\log a_{2}^{\prime} .
$$


On peut convenir que l'accent désigne l'état de référence. L'expérience donne une liste de valeurs de $E$ en fonction de $x_{2}$. On écrit alors (9) sous la forme

$$
\log \left(\frac{a_{2}}{x_{2}}\right)=\left(-\frac{E}{0,058 \mathrm{I} 6}-\log r_{2}\right)+\log \iota_{2}^{\prime} . \quad\left(y^{\prime}\right)
$$

On porte alors en ordonnée la parenthèse du deuxième membre, connue expérimentalement, en

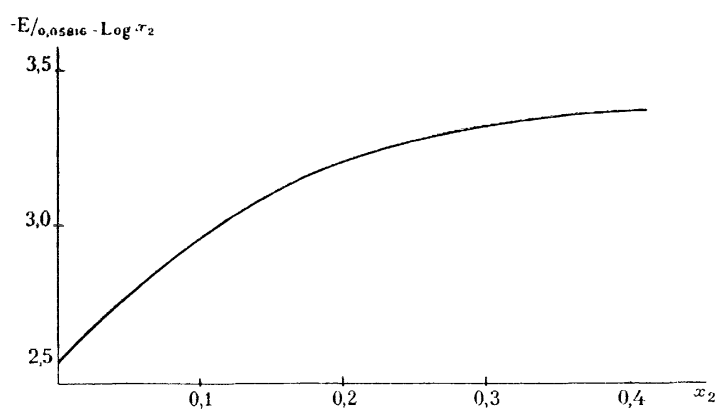

Fig. 5.

fonction de $x_{2}$ en abscisse, comme l'indique la figure 5 et l'on extrapole jusqu'à $x_{2}=0$. Si l'on remarque qu'en solution infiniment diluée, $a_{2} \rightarrow x_{2}$ ou $\log \left(\frac{x_{2}}{a_{2}}\right)->0$, l'ordonnée à l'origine est simplement $-\log a_{2}$, ce qui détermine l'activité dans. l'amalgame de référence. Connaissant cette valeur, on calcule facilement $a_{2}$ et $\gamma_{2}$ pour toute valeur de $x_{2}$. Le Tableau IV, où $0,003 \curvearrowright 6$ est la concentration de référence, donne les valeurs ainsi déduites par Lewis et Randall des mesures de Richards.

TaBleau IV.

\begin{tabular}{|c|c|c|c|c|}
\hline$x_{\Omega}$ & $-E$ & $\begin{array}{l}-\frac{E}{0,05816} \\
-\log x_{2}\end{array}$ & $\mu_{1 .}$. & $\gamma_{2 .}$. \\
\hline o & $-\infty$ & 2,4689 & o & I \\
\hline$o, 00326$ & o & 2,4869 & 0,0034 & I, 042 \\
\hline o,o1675 & 0,04555 & $2,559^{2}$ & 0,0206 & I , $23 \mathrm{I}$ \\
\hline$o, 03723$ & 0,07194 & 2,6660 & 0,0586 & I, 574 \\
\hline$o, o 4856$ & 0,0817 & 2,7184 & 0,0864 & I , 776 \\
\hline$o, o 986$ & o, I I I I 2 & 2,9177 & 0,2772 & $2,8 \mathrm{I} \mathbf{I}$ \\
\hline$o, \mathbf{1} 680$ & $0, \mathbf{1} 355$ & 3,1045 & $o, 7259$ & $4,3 \cdot 1$ \\
\hline 0,2074 & $o, 143 \mathrm{I}$ & $3, x>80$ & $\mathbf{I}, 06 \mathbf{I}$ & 5, I I \\
\hline o, 336 I & $o, \mathrm{I} 653$ & 3,3159 & 2,363 & 7,031 \\
\hline$o$, 伿和 & 0,1735 & 3,356 & 3,268 & 7,707 \\
\hline 0,428 (satt) & $0,1738-$ & 3,358 & $3,3 \mathrm{I} 6$ & $7,-75$ \\
\hline
\end{tabular}

On remarquera dans le tableau comment $\gamma_{2}$ s'éloigne rapidement de l'unité même pour des concentrations très faibles.
La méthode donne ici l'activité du. corps dissous; la pression de vapeur nous donne au contraire directement celle du solvant. On peut passer de l'une à l'autre par la méthode que nous avons indiquée. La formule de Gibbs s'applique toujours en effet sous la forme

$$
x_{1} d \ln c_{1}+x_{2} d \ln r_{2}=0 .
$$

Supposons qu'on veuille par exemple obtenir l'activité du mercure dans les amalgames précédents. On remarque que $\mathrm{d} x_{1}=-\mathrm{d} x_{2}$, donc (10) peut s'écrire :

$$
\mathrm{d} \ln \left(\frac{a_{1}}{x_{1}}\right)=-x_{x_{1}}^{x_{2}} \mathrm{~d} \ln \left(\frac{a_{2}}{x_{2}}\right),
$$

ou finalement

$$
\mathrm{d} \ln \dddot{\prime}_{1}=-\frac{x_{2}}{x_{1}} \mathrm{~d} \ln \% 2 .
$$

Pour calculer $\gamma_{1}$, on construira la courbe de $x_{2}$ en ionction de $\ln \gamma_{2}$ et l'on déterminera l'aire comprise sous la courbe à partir de $\gamma_{2}=\mathrm{I}$ correspondant

\begin{tabular}{|c|c|c|c|}
\hline$X_{\mathrm{y}}$. & $\frac{r_{2}}{x_{1}}$. & $\because_{1}^{\prime}$. & $\gamma_{1}$. \\
\hline 0 & 0 & 1 & I \\
\hline $0,00 j$ & 0,005 & 1,06 & 0.9998 \\
\hline 0, or & o,OIOI & I, Ij & $o, 999$ \\
\hline 0,05 & ; & 1.80 & 0,986 \\
\hline $0, I$ & 0,111 & ', & $o, 950$ \\
\hline 0,2 & 0,950 & $4,9^{8}$ & $o, 866$ \\
\hline$o, 3$ & 0,428 & 6,60 & 0,790 \\
\hline 0,4 & $0,66-$ & 7,57 & 0,734 \\
\hline o, 5 & 1,00 & 7,98 & o, ,o' \\
\hline
\end{tabular}
à la dilution infinie. On obtient ainsi les nombres du Tableau V.

Tableav $V$.

Nous retrouverons ce genre de calcul dans le paragraphe suivant.

Activité déduite des mesures CRyoscopiques. - Nous tenons enfin à traiter, au moins sommairement, cette question, pour bien montrer que, pendant des années, les physicochimistes ont employé des méthodes "élémentaires " qui n'avaient aucune valeur.

Lewis, ayant à traiter de l'équilibre solide-liquide, choisit un état de référence. Pour le liquide, on prend l'état pur; l'activité du liquide est $\mathrm{I}$ dans cet état; son enthalpie relative est zéro. Le solide possède alors une activité $a$ et une enthalpie $\Delta H$ égale à la chaleur de solidification. La relation (14) (exposé I) donne de suite

$$
\frac{\mathrm{d} \ln a_{s}}{\mathrm{~d} T}=-\frac{\Delta H}{R T^{2}} .
$$


On développe ensuite $\Delta H$ en série dans le voisinage du point de fusion. Pour cela, on pose

$$
r-T_{1}=0 \quad \text { et } \quad \mathrm{d} T=-\mathrm{d} \theta \text {. }
$$

En appelant $\Delta H_{0}$ la chaleur de solidification au P. F. et $\Delta C_{\mu}$, la différence des capacités calorifiques du solide et du liquide, on a, à la température $T$,

$$
\Delta I=\Delta I_{0}-\theta \Delta C_{\mu} .
$$

On substitue (14) et (15) dans (13) et l'on développe par rapport aux puissances de $\theta$; on obtient ainsi la série

$$
\begin{aligned}
\mathrm{d} \ln a_{1}=\frac{\mathrm{d} \theta}{R T^{2}}\left[\Delta H_{0}\right. & +\left(\frac{2 \Delta H_{0}}{T_{f}}-\Delta C_{p}\right) \theta \\
& \left.+\left(\frac{3 \Delta H_{0}}{T_{f}^{2}}-\frac{2 \Delta C_{p}}{T_{f}}\right) 0^{2}+\ldots\right] .
\end{aligned}
$$

On intègre ensuite à partir de $\theta=0$, en remarquant que, à cette température, il y a équilibre entre le solide et le liquide, donc $a_{s}=a_{\iota}=\mathrm{I}$. Il vient

$$
\ln a_{s}=\frac{\mathrm{I}}{R T_{f}^{2}}\left[\Delta H_{0} \theta+\left(\frac{\Delta H_{0}}{T_{f}}-\frac{\Delta C_{p}}{2}\right) \theta^{2}+\ldots\right] \text {. }
$$

Pour la glace et l'eau par exemple, gardant seulement le terme en $\theta$, on a $T_{j}=273, \mathrm{I} \Delta H_{0}=-\mathrm{I}_{4} 38$ et $\Delta C=-9$, ce qui donne

$$
\mathrm{d} \ln a_{s}=-(\dot{o}, 009696+0,0000103 \theta) \mathrm{d} \theta \text {. }
$$

C'est cette équation qui sert de base aux calculs des résultats cryoscopiques.

On peut remarquer que, pour une composition donnée d'une solution aqueuse, il existe un abaissement déterminé du point de fusion, de sorte que, dans (18), $\theta$ est fonction de la molarité par exemple. $\mathrm{Au}$ point de fusion dans la solution, l'activité de l'eau en solution est égale à celle de la glace, d'après la convention ci-dessus. Donc, si l'indice $\mathrm{I}$ se rapporte au solvant, on a

$$
\mathrm{d} \ln a_{1}=-(0,009696+0,0000 \operatorname{Io} 30) \mathrm{d} \theta
$$

et en intégrant

$$
\ln a_{1}=-0,0096960-0,000005102 .
$$

Dans l'équation (20), $a_{1}$ est l'activité de l'eau au point d'apparition de la glace. En négligeant la variation de l'enthalpie avec la concentration, c'est-à-dire en négligeant la chaleur de dilution, on peut supposer que $a_{1}$, pour une concentration donnéc, est indépendant de la température. Cela étant, on considérera 0 dans les équations précédentes comme une propriété de la solution, fonction de la concentration et, pour calculer l'activité du corps dissous, on emploiera l'équation (10) ci-dessus. On en tire

$$
\mathrm{d} \ln a_{2}=-\frac{55,5 \mathrm{I}}{m}(-0,009696 \theta-0.0000103 \theta) \mathrm{d} \theta .(21)
$$

Dans cette équation, quand $\theta \rightarrow 0$, on obtient l'abaissement cryoscopique limite

$$
\frac{d \theta}{d m}=55,51 \times 0,009696=1 \text {. } 8 \text { is. }
$$

Soit $\lambda$ ce nombre. En introduisant ce nombre, l'équation (21) prend la forme

$$
\mathrm{d} \ln a_{2}=\frac{\mathrm{d} \theta}{\lambda m}+0.00057 \theta \frac{\mathrm{d} \theta}{m} .
$$

Pour intégrer cette équation, Lewis pose

$$
j=\mathrm{I}-\frac{0}{i m !}
$$

on en lire

$$
\frac{\mathrm{d} \theta}{i, m}=(\mathrm{r}-j) \mathrm{d} \ln m-\mathrm{d} j
$$

et, en substituant dans (22),

$$
\mathrm{d} \ln \left(\frac{a_{2}}{m}\right)=-j \mathrm{~d} \ln \ln -\mathrm{d} j+0.00057 \theta \frac{\mathrm{d} \theta}{m} .
$$

On intègre entre les molarités $\theta$ et $m$; la constante

\begin{tabular}{|c|c|c|c|c|c|c|c|}
\hline ג & 0 & $\frac{\theta}{m}$. & $-j$ & $-\frac{i}{m}$ & A. & B. & $\frac{a_{2}}{m}$. \\
\hline$o, \mathrm{I}$ & $\mathrm{o}, \mathrm{I} 863$ & I, 863 & $0,002 j$ & $0,02 j$ & 0,003 & 0,0002 & I,, 006 \\
\hline 0,2 & 0,3734 & I, 867 & 0,005 & 0,025 & 0,006 & 0,0005 & I, OI 2 \\
\hline$o, j$ & 0,9430 & I, 886 & 0,015 & 0,030 & 0,016 & 0,0009 & $\mathrm{I}, 032$ \\
\hline $1, O$ & $I, 918$ & I, 918 & 0,032 & 0,032 & $0,03 \mathrm{I}$ & 0,0021 & I,, 068 \\
\hline 2,0 & 3,933 & I. 966 & o, 058 & 0.029 & 0,062 & 0,0041 & $\mathbf{I}, \mathbf{1} 32$ \\
\hline 5.0 & 10,58 & 2.116 & 0.139 & $0,0,8$ & 0,148 & 0,0120 & I, 348 \\
\hline
\end{tabular}
d'intégration est nulle, $a_{2}$ tendant vers $m$ et $j$ vers zéro quand la dilution est infinie. On a finalement

$$
\begin{aligned}
\ln \left(\frac{a_{2}}{m}\right) & =\int_{0}^{m}-j \mathrm{~d} \ln m-j+\int_{0}^{. \prime \prime} 0,00057 \frac{\theta}{m} \mathrm{~d} \theta \\
& =A-j+B .
\end{aligned}
$$

Lewis a par exemple traité à l'aide de cette équation les résultats relatifs aux solutions aqueuses de glycérine. L'intégrale A se déduit de la courbe - en fonction de $m$; l'intégrale $B$ de la courbe $\frac{0}{m}$ en fonction de $\theta$. Cette dernière courbe a été précisément considérée par Raoult, pour obtenir son "ordonnée à l'origine ". Les calculs de Lewis ont donné, pour la glycérine, les résultats suivants (Tableau VI).

TABLEAU VI.

La dernière colonne donne le coefficient d'activité (rapporté à la molarité).

En solution assez étendue, $\frac{j}{m}$ est à peu près 
constant. Si l'on admet cette régularité, on obtient l'équation approchée

$$
2,303 \log \left(\frac{a_{\cdot 2}}{m}\right)=-2 j
$$

qui suffit généralement pour traiter les mesures assez peu précises que renferme la littérature des solutions non électrolytiques. On trouvera dans Lewis et Randall les compléments relatifs à l'ébullioscopie.

C. Courbes et formules pour le coefficient d'activité. - Nous allons supposer de nouveau que nous nous occupons de mélanges de liquides

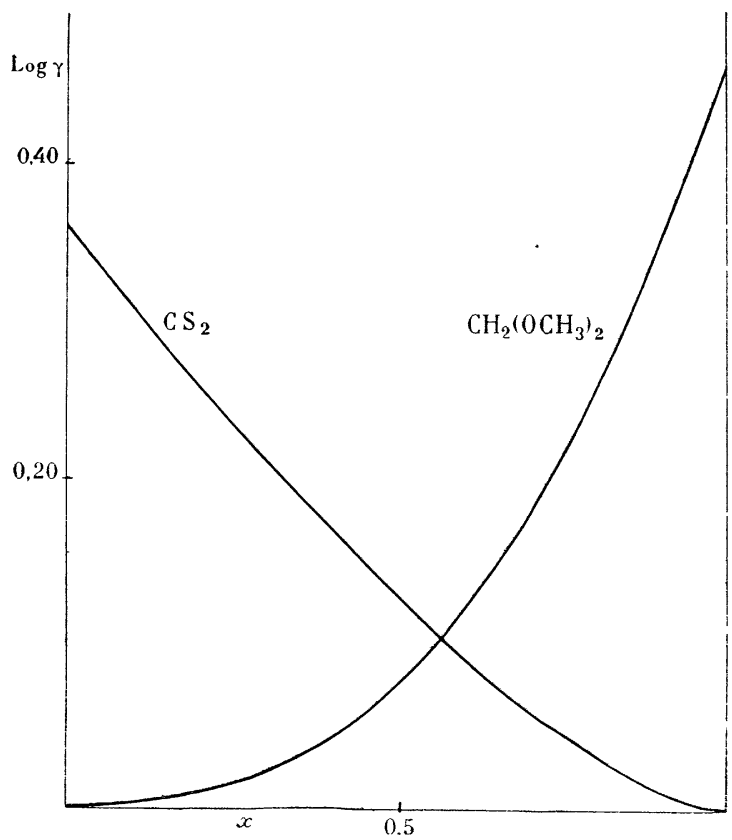

Fig. 6.

Le coefficient d'activité du $n^{0} 1$ est égal à I pour le liquide pur, $\log \gamma_{1}$ est nul pour $x_{2}=0$; c'est donc une fonction de $x_{2}$ qui s'annule pour $x_{2}=0$. De même pour $\log \gamma_{2}$. La figure $2 / 4$ représente les valeurs de $\log \gamma_{1}$ en fonction de $x_{2}$ et de même pour $\log \gamma_{2}$, pour les mélanges sulfure de carboneméthylal. On voit que ces courbes ont une allure parabolique suggérant un développement suivant les puissances croissantes des $(x)$. On peut donc poser

$$
\left.\begin{array}{l}
\log \gamma_{1}=A_{1} x_{2}+\frac{B_{1}}{2} x_{2}^{2}+\frac{C_{1}}{3} x_{2}^{3}+\ldots, \\
\log \gamma_{2}=A_{2} x_{1}+\frac{B_{2}}{2} x_{1}^{2}+\frac{C_{2}}{3} x_{1}^{3}+\ldots
\end{array}\right\}
$$

Nous avons déjà dit que la formule de Duhem-
Margules établit une relation entre les pressions partielles, donc en définitive entre les coefficients d'activité; c'est la relation déjà vue plus haut et que nous écrirons

$$
x_{1}\left(\frac{\mathrm{d} \ln \check{r}_{1}}{\mathrm{~d} x_{1}}\right)_{r_{2}}+x_{2}\left(\frac{\mathrm{d} \ln \check{r}_{2}}{\mathrm{~d} x_{1}}\right)_{x_{2}}=0 .
$$

$\mathrm{Si}$ on limite les développements (26) aux quatre premiers termes, on tire de suite de la formule de Duhem-Margules les relations suivantes :

$$
A_{1}=A_{2}=0, \quad B_{2}=B_{1}+C_{1}+D_{1}, \quad \ldots
$$

Il est à retenir d'abord de ces relations que les développements n'ont pas de terme en $x_{1}$ et $x_{2}$. Cela prouve que, dans un mélange réel, la loi de Raoult est vérifiée, non seulement pour $x=0$, mais pour une série de valeurs de $x$ voisines de zéro. Ce résultat correspond au fait que, dans les courbes de pression partielle, la courbe est tangente à la droite de Raoult vers $x=0$. Autrement dit encore, la loi de Raoult est une loi-limite indépendante du corps dissous, pour un solvant donné $\left(x_{2}\right.$ ne figure pas au premier degré dans $\left.\log \gamma_{1}\right)$.

Comme cas particulier des formules (26), nous utiliserons un développement du $2^{\mathrm{e}}$ degré

$$
\log \varkappa_{1}=B x_{2}^{2} \quad \text { et } \quad \log \Upsilon_{2}=B x_{1}^{2} .
$$

Ces développements semblent convenir pour les solutions de $\mathrm{Tl}$ et de $\mathrm{Cd}$ dans l'étain; dans ce cas, le graphique de $\log \gamma$ en fonction de $x_{2}$ est une droite. Van Laar a proposé une formule du type

$$
\log \gamma_{1}=\frac{\alpha_{1}}{\left(\mathrm{I}+\beta_{1} r_{1}\right)^{2}}
$$

et l'expression analogue pour $\gamma_{2}$; les $r$ sont le rapport $\frac{x_{1}}{x_{0}}$ et son inverse. Les coefficients $(\alpha)$ et $(\beta)$ sont également reliés par l'équation de DuhemMargules. D'après Hildebrand, pour les amalgames de $\mathrm{Cd}$ et $\mathrm{Sn}$ à $323^{\circ} \mathrm{C}$, le coefficient d'activité de $\mathrm{Hg}$ est bien représenté par des équations de ce type; on aurait respectivement $\alpha_{1}=-1,40$ et 0.22 et $\beta_{1}=1,90$ et 0,26 .

D. Effet de la température sur les déviations de la loi de Raoult. - L'élévation de $T$ a généralement pour effet de rendre les mélanges plus idéaux. C'est ce que montre la figure 7 qui représente, d'après Schulze [ro], la pression totale pour le système chloroforme-acétone à $30^{\circ}$ et $90^{\circ} \mathrm{C}$. L'échelle de gauche, en millimètre de $\mathrm{Hg}$, celle de droite en kilogramme par centimètre carré sont relatives aux deux températures. La déviation est négative dans les deux cas, mais moins forte à $90^{\circ}$ qu'à $30^{\circ}$.

Inversement, l'abaissement de la température rend le mélange de moins en moins idéal. Cet effet est particulièrement marqué dans les mélanges qui 
se séparent en deux couches liquides à basse température.

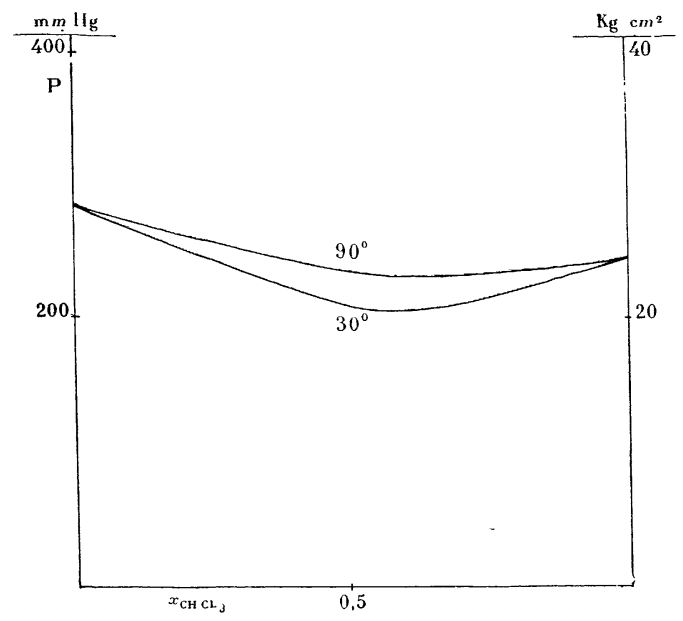

Fig. 7.

E. Apparition de deux couches liquides. La discussion de cette question est relativement simple si l'on admet que le développement de $\log \%$ est réduit au terme en $x^{2}$. Nous verrons, plus tard que, d'après Hildebrand, ce cas est assez général pour les mélanges dont les composants ont des volumes molaires assez voisins. Si $\log _{10} \varkappa=A x^{2}$, on obtiendra des déviations croissantes en augmentant $A$. Nous avons reproduit [ir] le calcul de l'activité du corps $\mathrm{n}^{\circ} 2$ pour divers $\left(x_{2}\right)$, en donnant à $A$ des valeurs croissantes; on trouve ainsi que la courbe $a_{2}=f\left(x_{2}\right)$ a la forme donnée par la figure 8 pour différentes valeurs de $A ; A=0$ correspond au cas idéal. Pour les grandes valeurs de $A$, la courbe a deux bosses et la partie médiane correspond à un état instable; le mélange correspondant se sépare en deux couches $P$ et $Q$. L'importance de ces deux couches dépend de la quantité de produit disponible. Si l'on ajoute au no 1 pur des quantités croissantes $d u n^{\circ} 2$, il y a d'abord simple dissolution; le point représentatif décrit l'arc de gauche de la courbe. Pour la composition $P$, apparaît la deuxième couche dont la quantité augmente, le volume de la première couche diminuant; pour une quantité suffisante du $\mathrm{n}^{\circ} 2$, la couche de composition Q subsiste seule et le point décrit ensuite la partie de droite de la courbe.

Quand la température augmente, les déviations sont moindres; il faut admettre que $A$ diminue; on arrive à la courbe marquée $A=0,9$ et le mélange homogène devient alors possible. Au-dessus d'une certaine température, il y a donc dissolution homogène des deux liquides; on dit que le couple de liquides possède une température critique de dissolution (T. C. S.); on aurait alors pour les deux composants $\frac{d x}{d a}=0$ et $\frac{d x^{2}}{d^{2} a}=0$. Ces deux relations, écrites pour $\log _{10} \because=A x$, donnent $x_{1}=x_{2}=0,5$, avec $A=\frac{0,434}{0, \tilde{j}}=0,868$. Cette valeur "critique ". de $A$ est bien voisine de $A=0,9$ qui donne la longue inflexion dans la courbe. Comme nous l'avons

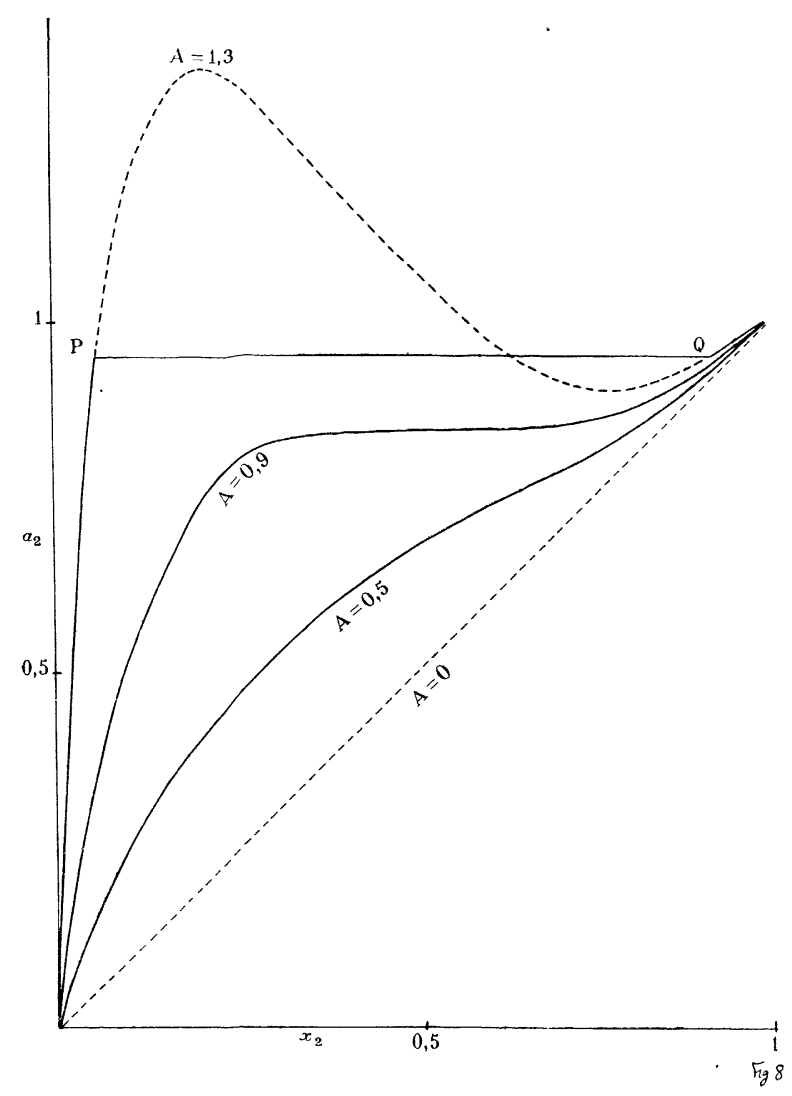

Fig. 8 .

remarqué ci-dessus, dès l'instant qu'on arrête le développement de $\log \gamma$ au terme en $x^{2}$, il y a symétrie entre les deux composants. Ce n'est pas tout à fait le cas en pratique, comme le montre la figure 9 qui donne la composition des mélanges en équilibre aux diverses températures pour le couple aniline-hexane (T. C. S. $60^{\circ}$ ).

T. C. S. SUPÉrieUre et infÉrieUre. - Dans ce qui précède, il a été question de mélanges semblables au mélange aniline-hexane, qui subissent la démixtion au-dessous d'une certaine température. On dit que ces mélanges possèdent une T.C. S. supérieure. C'est le cas aussi pour les mélanges eau-phénol, hexane-nitrobenzène, pétrole-alcool, etc. Ces mélanges ont été beaucoup étudiés à toutes sortes de points de vue. Ils offrent en effet une image facilement 
accessible des phénomènes de vaporisation critique auxquels ils ressemblent beaucoup.

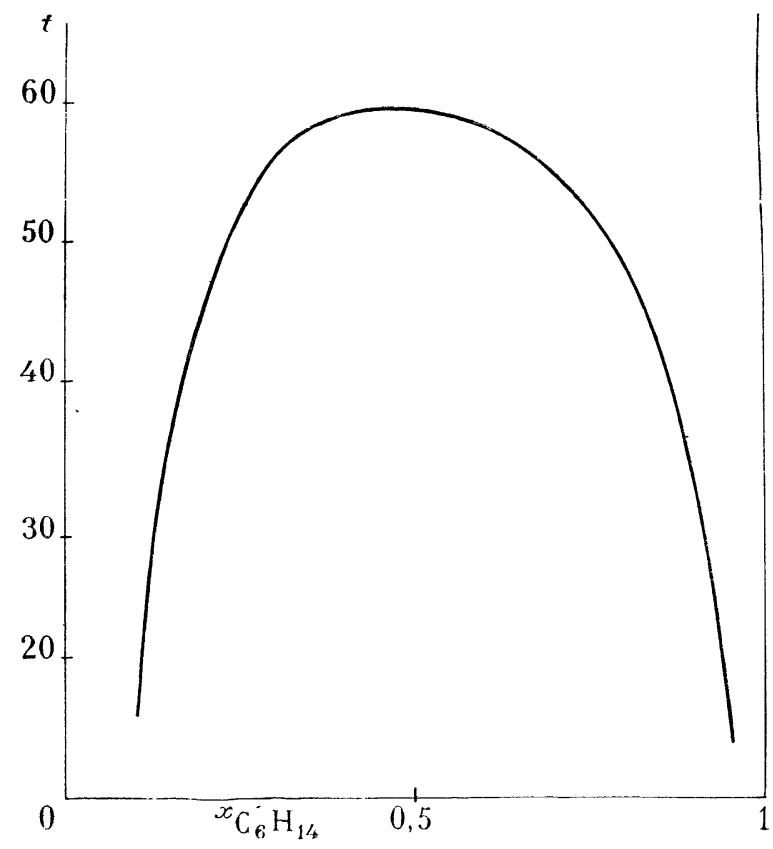

Fig. 9.

Quand la température s'élève, les deux liquides superposés tendent l'un vers l'autre; l'indice de réfraction (M1le Schlegel) [ [13], la densité, la tension superficielle (Antonoff) [ra], etc., deviennent identiques. On retrouve même l'opalescence critique qu'on a également étudiée, tant au point de vue expérimental qu'au point de vue théorique (Rocard et Andant [r5], Rousset [г6]). On a même retrouvé sur les courbes analogues à celles de la figure 9 la loi du diamètre rectiligne de Cailletet et Mathias; la demi-somme des concentrations (exprimées en titre) correspondant aux solutions conjuguées est une fonction à peu près linéaire de $T$.

Pour comprendre complètement les phénomènes de démixtion, il faut considérer l'équilibre des deux liquides avec leur vapeur commune, comme l'a montré Kuenen.

Le système, comprenant deux composants et trois phases est univariant, d'après la règle des phases, d'où une composition complètement déterminée dès qu'on fixe la température. Si celle-ci varie, les compositions des deux phases liquides et celle de la vapeur changent; à la T. C. S. la phase vapeur n'a pas la composition commune aux deux liquides.

L'existence d'une T.C.S. supérieure est très fréquente, mais pas absolument générale. Certains mélanges (eau-nicotine; glycérine-m-toluidine) pos- sèdent un domaine de démixtion complètement fermé, donc à la fois une T. C. S. supérieure et une T. C. S. inférieure. La figure ro représente, dans le diagramme $(T, x)$, le domaine de démixtion pour le mélange glycérine- $m$-toluidine.

Pour certains couples, on ne peut réaliser aux températures accessibles que la partie inférieure de la courbe; ils possèdent seulement une T. C. S. inférieure. Exemples : eau-triéthylamine; $\mathrm{CO}_{2}$-nitrohenzène; $o$-nitrophénolnitrobenzène. Quand on augmente la température, l'une des phases liquides se vaporise complètement et il ne reste en présence qu'un liquide riche en le composant le moins volatil et une vapeur riche en le composant le plus volatil.

Il existe enfin quelques couples où la solubilité mutuelle est toujours trop faible pour donner un mélange homogène; il n'y a aucune T. C. S. (mélange méthane-alcool).

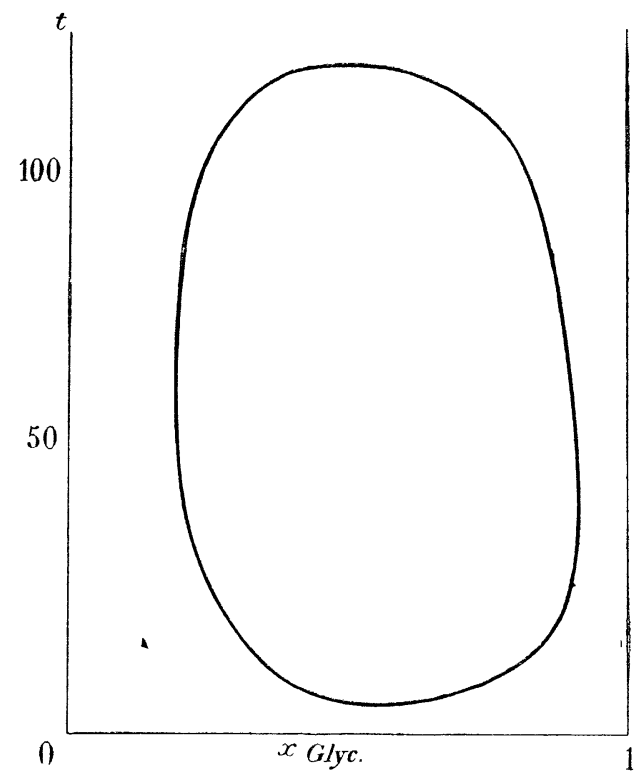

Fig. 10 .

Les calculs effectués ci-dessus sur l'équation $\log \gamma_{2}=A x_{1}$ ne peuvent plus s'appliquer pour une T. C. S. inférieure, à moins de supposer que $A$ augmente cette fois quand $T$ augmente. Il est possible aussi que la formule soit plus compliquée. On vérifiera qu'une formule du type $\log \gamma=A x^{2}+B x^{3}$ donne par exemple des ( () inférieurs à l'unité quand $A$ est négatif et $B$ positif, pour certaines valeurs de $A$ et $B$. Pour trouver des $(\gamma)$ inférieurs à l'unité, il faut supposer $A<0$; avec un seul terme, la courbe de $a_{2}$ n'a dans ce cas aucune singularité. Avec deux termes $(A<0$ et $B>0)$, on retrouve des inflexions, 
mais cette fois au-dessous de la droite de Raoult. Exemple : $A=-3, B=+4$; on obtient une courbe à deux bosses.
Dans un autre exposé, nous parlerons des raisons invoquées pour expliquer les discordances avec la loi de Raoult.

\section{BIBLIOGRAPHIE.}

[1] Schröder, Zeit. f. phys. Chem., i893, 11. p. 449.

[2] Le Chatelier, C. R. Acad. Sc., 1894, 118, p. 638.

[3] Washburn et Read, Proc. Nat. Acad. Sc., i 9 1 5, 1, p. I91.

[4] Kremann, Monatsh. f. Chem., г904, 25, p. т 2 г 5 et 1 9 I 6,37 , p. 59 .

[5] Van LaAr, Kon. Akad. Wet, Amst., iguG 711;

[6] P. Joв, Ann. Chim., I 928 , 9, p. 113.

[7] Hildebrand et Eastman, J. Ann. Chem. Soc., i915, 37, p. 2452 .

[8] Lewis et StoRCh, ibid., 1917, 39, p. 2544.
[9] Richands el Daniels, ibid., 1919, 41, p. 1732.

[10] Schulze, Zeits. f. phys, Chem., ig19, 93, p. 368.

[ir] E. Darmois, E'état liquide de la matière, Albin Michel, Paris, I 94.3.

[12] Timarernians, Les solutions concentrées, Masson, Paris, 1936.

[13] Nile Schleger., J. Chim. Phys, i $934, \mathbf{3 1}, 668$.

[14] Aхтолот, Phil. Mag., 1926, 1, , г г et suivants.

[i5] Rocard et ANodint, Soc. fr. Phys., i $925,30$.

[16] Rousset, C. R., I $934,198,2$ I 52 et 199, 716 . 